\title{
Dear Readers!
}

DOI: $10.1134 / \mathrm{S} 1063771010060011$

Herewith we would like to call your attention to this and next issues of Acoustical Physics dedicated to the state of the art of "WAVE PROCESSES IN MEDIA WITH MICRO- AND NANOSTRUCTURE."

Recently, two famous dates passed that are directly related to this problem. The first was the centennial of the appearance of continuum theory, taking into account internal (rotational) degrees of freedom (Cosserat E. and Cosserat F., Theorie des corps deformable, Paris: Hermann, 1909). The second date is the 50 -year anniversary of the lecture "There's Plenty of Room at the Bottom," read by Richard Feynman in December 1959 before the American Physical Society. In his lecture, Feynman indicated the possibility of using individual atoms and molecules as "building elements" to create new objects and materials with unique properties. The first event was the beginning of the theory of media with microstructure, and the second is considered by many to be the birthdate of nanotechnology. At present, these are two actively developing areas with a large array of physical and practical results. So, it has been possible to describe many properties of biological tissues, geological species, and other natural objects via models of media with microstructure. On the other hand, construction of new structures from artificial cells with a certain internal device has already led to the creation of superhard materials, media with heretofore unseen wave structures, and other wonderful results. Note that both in understanding the behavior and in synthesizing new devices and materials of this type, acoustic processes and their interaction with other physical fields play an important role.

September 21-23, 2009, the First All-Russia conference "Problems of Mechanics and Acoustics of Media with Micro- and Nanostructure: NANOMEKH 2009" was held, based at Nizhni Novgorod's Alekseev Technical University. Nearly 100 reports were presented. This issue of Acoustical Physics is based on selected reports from the acoustical section of NANOMEKH 2009 , supplemented by a number of other works on this topic.

Part of this issue's papers shine light on results on the theory of linear and nonlinear waves in layered media, crystal lattices, media with inclusions of different physical nature, and classical Cosserat media. There are also three review articles on Rayleigh-type elastic waves in layered compositions, as well as of waves in magnetic nanoliquids and microinhomogeneous media. Special attention is focused on nonlinear effects in such media and structures. More than a quarter of the papers are devoted to experimental methods using acoustic waves to evaluate the parameters of the internal structure of materials. A notable place is given to the problem of acoustic wave interaction with magnetic and electric fields and the problem of formation of internal structure under the action of acoustic fields.

These papers adequately reflect the level of Russian research in many directions related to the creation of new materials with micro- and nanostructure. Acoustical Physics is intent on devoting much attention to this problem in the future.

Compilers of the issue: Yu.I. Bobrovnitskii and A.I. Potapov ${ }^{\dagger}$

Translated by $A$. Carpenter 